\title{
MÚSICA Y COMPETENCIAS EMOCIONALES: POSIBLES IMPLICACIONES PARA LA MEJORA DE LA EDUCACIÓN MUSICAL
}

\author{
Emilia Ángeles Campayo Muñoz \\ Universidad Jaume I (Castellón) \\ emicampayo@gmail.com
}

\author{
Alberto Cabedo Mas \\ Universidad Jaume I (Castellón) \\ cabedoa@uji.es
}

\begin{abstract}
El presente artículo estudia las relaciones existentes entre la música y el desarrollo de las competencias emocionales. Se explora la capacidad de la música para inducir emociones en el individuo. Se examina la predisposición natural del ser humano hacia la música, así como los efectos que la práctica instrumental genera en el cerebro. Se explican los efectos que la música produce en el comportamiento del oyente e intérpretes. Finalmente, se sugiere cómo la educación musical podría ayudar al desarrollo de las competencias emocionales y las posibles implicaciones que esto tiene para la mejora de la educación musical.

This paper reviews the relationships between music and the development of emotional skills. It studies the power of music to induce emotions on the individual and addresses the difficulties to measure resultant emotional responses. It examines the human's natural predisposition to music as well as the effects that musical practice generates on brain. Finally, it explains the effects music causes on both listeners' and musicians' behaviour. It suggests how music education could help to the development of emotional skills and its possible implications for the enhancement of music education.
\end{abstract}

Palabras clave: Música, inteligencia emocional, competencias emocionales, educación musical, neurociencia.

\section{INTRODUCCIÓN}

Las emociones determinan en gran medida la experiencia de vida, son parte inherente de la naturaleza humana, e influyen de manera directa en la toma de decisiones y, por tanto, en las acciones cotidianas. Damasio (2001) afirma que no se logra un conocimiento acertado del funcionamiento del cerebro y de la mente en el campo de la ciencia sin tener en cuenta la emoción como uno de los componentes básicos de los mismos. La emoción juega un papel importante en la biorregulación, la supervivencia y la memoria. El conocimiento y correcta conducción de nuestras propias emociones es fundamental para conseguir una realización personal y profesional (GOLEMAN 1996, 1998). La inteligencia emocional es la habilidad de ser consciente de nuestras emociones y de saber gestionarlas adecuadamente. Como habilidad, la inteligencia emocional puede aprenderse para ser mejorada. De este constructo, se entiende el concepto de competencia emocional como la capacidad de la persona para aplicar o poner en práctica esta inteligencia emocional en su cotidianidad (BISQUERRA \& PÉREZ, 2007).

El concepto de inteligencia emocional es relativamente reciente. Sin embargo, la preocupación por el mundo de las emociones y su influencia en nuestra conducta es un tema tratado desde la Antigüedad. Platón y Aristóteles muestran interés hacia el área de estudio, aunque es este último quien realiza, de forma pionera, uno de los primeros trabajos en profundidad acerca de las emociones. Según palabras de Kosman (1980), una de las ideas aristotélicas con respecto a lo que 
se entiende por inteligencia emocional en la actualidad es que "el arte de vivir de manera apropiada incluye el arte de sentir bien, como disciplina correlativa al actuar bien" (p. 105).

El concepto de inteligencia surge a finales del siglo XIX. En sus inicios, este término se fundamenta en aspectos puramente cognitivos, aunque algunos investigadores pronto asocian el concepto de inteligencia a factores más allá de los meramente cognitivos (CHERNISS, 2000). Alfred Binet, a comienzos del siglo XX, es uno de los pioneros en afirmar que el éxito escolar no reside de manera exclusiva en el cociente intelectual (CI) de la persona (MOLERO, SAIZ \& ESTEBAN, 1998). A principios de la década de los años 20, Edward Thorndike propone incluir diferentes tipos de inteligencias: la social, mecánica y abstracta (TRUJILlo \& RIVAS, 2005). Sin embargo, estas aportaciones no tienen grandes repercusiones en el desarrollo del concepto de inteligencia hasta el año 1983, cuando Gardner (1983) en su estudio Frames of Mind, le confiere un nuevo componente humanista. En oposición a visiones anteriores del concepto de inteligencia, considerado como un aspecto estático durante toda la vida de una persona, Gardner la define como una capacidad, lo cual implica una posibilidad de cambio o evolución. Según el autor, la inteligencia está formada por un conjunto de otras inteligencias. En un principio, el autor define siete tipos de inteligencias: a) inteligencia lógica-matemática, b) inteligencia verbal-lingüística, c) inteligencia espacial, d) inteligencia musical, e) inteligencia corporal-cinestésica, f) inteligencia intrapersonal, g) inteligencia interpersonal. Con posterioridad, añade la inteligencia naturalista y la inteligencia existencial. Posteriormente, en 1990, y con base en los conceptos de inteligencia intrapersonal e interpersonal de Gardner, Salovey y Mayer (1990) definen el concepto de inteligencia emocional. Este nuevo concepto genera un creciente interés en la literatura científica, y grandes índices de popularidad tras la publicación de Emotional Intelligence, de Daniel Goleman (1995). Salovey y Mayer (1997) establecen cuatro dimensiones interrelacionadas asociadas al concepto de inteligencia emocional: a) percepción de las propias emociones, b) uso de las emociones, c) comprensión de las emociones y d) control de las emociones en las relaciones. Según Bisquerra y Pérez (2007), el constructo de inteligencia emocional es hipotético, y a día de hoy, en el campo de la psicología, su existencia está todavía cuestionada.

Con base en lo anteriormente expuesto, la inteligencia emocional se define como una habilidad que comprende la emoción y razón, y que posibilita aprender a entender y gestionar las emociones con el fin de favorecer el bienestar personal (FERNANDEZ-BERROCAL \& EXTREMERA-PACHECO, 2005). Numerosos autores han investigado este campo creando modelos que, si bien en esencia comparten los mismos principios, presentan diferentes matices. Estos se pueden agrupar en a) modelo de habilidades y b) modelo mixto. El primero se centra en un conjunto de capacidades mentales que permiten utilizar la información que se obtiene a través de las emociones para mejorar los procesos cognitivos (FernándeZ-BERrocal, Berrios-Martos, Extremera \& Augusto, 2012). El segundo modelo combina las habilidades mentales del primer modelo con competencias emocionales y rasgos estables de comportamiento y personalidad. Este modelo tiende a realizar afirmaciones acerca de los beneficios del desarrollo de las competencias emocionales en la persona (COBB \& MAYER, 2000).

Las competencias emocionales se derivan de la inteligencia emocional. Bisquerra y Pérez (2007), las definen como "un constructo amplio que incluye diversos procesos y provoca una variedad de consecuencias" (p. 5). Según los autores, a diferencia de la inteligencia emocional, "la competencia emocional pone el énfasis en la interacción entre persona y ambiente, y como consecuencia confiere más importancia al aprendizaje y desarrollo. Por tanto tiene unas aplicaciones educativas inmediatas" (p. 12). Los autores cuyos modelos de competencias emocionales gozan de mayor reconocimiento científico y aplicación, incluyen, entre otros, a Salovey y Sluyter (1997), Saarni (1997, 2000) o Goleman, Boyatzis y Mckee (2002). Dichos modelos están constituidos por diferentes dimensiones de la inteligencia emocional de las cuales, a su vez, se derivan una serie de competencias emocionales. 
En España, desde 1997, el GROP (Grup de Recerca en Orientació Psicopedagògica), integrado por profesores de la Universitat de Barcelona y de la Universitat de Lleida, ha realizado una labor de investigación intensa acerca de las competencias emocionales aplicadas al ámbito de la educación. El GROP ha creado su propio modelo de competencias emocionales, en continuo proceso de actualización. Según este modelo, las competencias emocionales se agrupan en cinco bloques, tal y como sugiere en el siguiente diagrama:

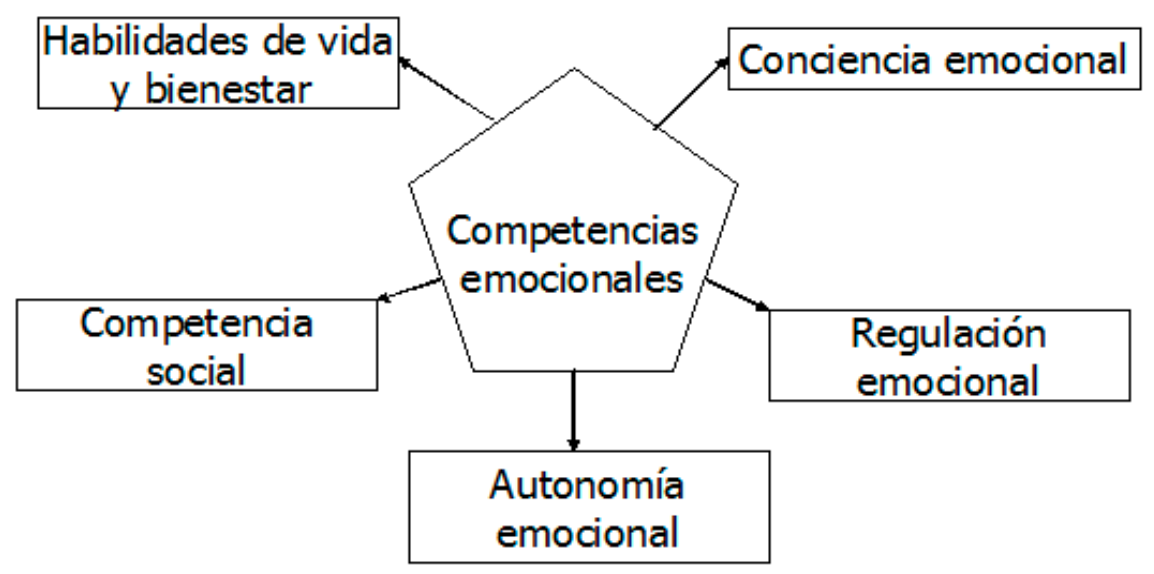

Figura 1: Modelo pentagonal del GROP de competencias emocionales (Bisquerra \& Pérez, 2007, p. 9)

El interés por implementar programas de educación emocional en diversos campos está justificado en base a la necesidad de las personas de desarrollar un mayor conocimiento y gestión emocional (BISQUERRA \& PÉREZ, 2007).

La música tiene una estrecha relación con las emociones (JIMÉNEZ, 2014; PELLITTERI, STERn \& NAKhutina, 1999; Robinson, 1995; SASTRe, 2014). Por ello, en el propósito de incluir la educación emocional en la formación global de las personas, la práctica musical tiene, sin duda, cabida. El objetivo de este estudio es revisar la fundamentación teórica del campo de estudio que relaciona la experiencia musical como medio idóneo para trabajar y desarrollar las competencias emocionales.

\section{MÉTODO}

El estudio se ha llevado a cabo a partir de una búsqueda bibliográfica en la que se han seleccionado aquellos artículos considerados como atingentes para dar respuesta al objetivo planteado. Con este fin, se han revisado las bases de datos electrónicas de Web of Science, Scopus y ERIC. Los descriptores usados, tanto de forma aislada como en diferentes combinaciones, fueron emotion, emotional intelligence, emotional skills y música. A esta búsqueda se le aplicaron los siguientes filtros iniciales: a) ámbitos de investigación, seleccionando aquellos que pertenecían a las ciencias sociales, b) áreas de investigación, centrándonos en los pertenecientes a las áreas de la psicología, neurociencia y música, c) tipo de documento, incluyendo únicamente revisiones y artículos, d) idioma, seleccionando aquellos escritos en lengua inglesa y española y e) período de la búsqueda, desde 1990 hasta la actualidad. Puesto que la inteligencia emocional no fue objeto de estudio hasta 1990, fue esta la fecha seleccionada como inicio de la búsqueda.

A esta primera selección se incluyeron nuevos artículos y libros derivados de la revisión de las referencias bibliográficas de aquellos artículos ya seleccionados. Al mismo tiempo, se incluyeron en la muestra algunos libros que se consideraron de interés por su relación indirecta entre música y competencias emocionales. No se encontraron libros que aborden este tema de forma directa. 
En una primera fase, el proceso de selección se realizó por los dos autores de manera independiente. Posteriormente, se llevó a cabo un estudio conjunto y consensuado de aquellos trabajos cuya inclusión planteaba dudas. Dada la escasa literatura existente en relación a la música y las competencias emocionales y/o la inteligencia emocional, se decidió incluir y clasificar también estudios que abordan temáticas relacionadas indirectamente con dicha literatura. Estas temáticas engloban investigaciones en torno a: a) música y emoción, b) música y neurociencia y c) música y conducta.

Un total de 28 textos fueron finalmente seleccionados y analizados. La información se categorizó de manera inductiva a partir de la naturaleza temática de los estudios. La siguiente tabla (Tabla 1) muestra las categorías y subcategorías que emergieron del análisis temático de los textos.

\begin{tabular}{|c|l|}
\hline Música y emoción & $\begin{array}{l}\text { Capacidad de la música para inducir emociones } \\
\text { Función de la música en la sociedad } \\
\text { Dificultad para medir las respuestas emocionales }\end{array}$ \\
\hline \multirow{2}{*}{ Música y neurociencia } & $\begin{array}{l}\text { Predisposición natural del ser humano hacia la música } \\
\text { Efectos de la práctica instrumental sobre el cerebro }\end{array}$ \\
\hline \multirow{2}{*}{ Música y conducta } & $\begin{array}{l}\text { En oyentes } \\
\text { En intérpretes }\end{array}$ \\
\hline
\end{tabular}

Tabla 1: Clasificación temática de los estudios

La Tabla 2 muestra los artículos que han sido incluidos en cada una de las categorías temáticas. Estos trabajos han tenido un gran impacto dentro de sus áreas de conocimiento y son, por ende, citados con frecuencia. Los textos han sido clasificados en estudios de investigación básica algunos de los cuales son revisiones de la literatura (rev) - e intervenciones; al mismo tiempo, se han clasificado textos que no provienen de publicaciones a través de artículos en revistas científicas, sino de libros, actas de congresos o tesis doctorales.

\begin{tabular}{|c|c|c|c|}
\hline & MÚSICA Y EMOCIÓN & $\begin{array}{c}\text { MÚSICA Y } \\
\text { NEUROCIENCIA }\end{array}$ & MÚSICA Y CONDUCTA \\
\hline $\begin{array}{l}\text { ARTÍCULOS DE } \\
\text { INVESTIGACIÓN } \\
\text { BÁSICA }\end{array}$ & $\begin{array}{ll}\text { - } & \text { García-Moreno, } 2011 \\
\text { - } & \text { Grabrielsson, } 2002 \\
\text { - } & \text { Gustems, 2001 } \\
& \text { Gustems \& Calderón, } \\
\text { - } & \text { Juslin, 2003 } \\
\text { - } & \text { Koelsch, 2010, 2015 } \\
\text { - } & \text { North \& Hargreaves, } \\
\text { - } & \text { Robinson, 1995 } \\
\text { - } & \text { Soria-Urios, Duque, \& } \\
\text { - } & \text { Vuilleumier \& Trost, } \\
& \text { 2015 } \\
\text { - } & \text { Zatorre, 2003 }\end{array}$ & $\begin{array}{ll}\text { - } & \text { Altenmuller, Gruhn, } \\
& \text { Parlitz, \& Liebert, } \\
& 2000 \\
\text { - } & \text { Collins, } 2013 \text { (rev.) } \\
\text { - } & \text { Gruhn, } 2005 \\
\text { - } & \text { Gustems Carnicer, } \\
& 2001 \\
\text { - } & \text { Hallam, } 2010 \text { (rev.) } \\
\text { - } & \text { Hodges, } 2010 \\
\text { - } & \text { Lacárcel, } 2003 \\
\text { - } & \text { Peretz, 2002 (rev.) } \\
\text { - } \quad \text { Perlovsky, 2010 } \\
\text { - Soria-Urios et al., } \\
\text { 2011 }\end{array}$ & $\begin{array}{ll}\text { - } & \text { Casas, } 2001 \\
\text { - } & \text { Chung, } 2010 \\
\text { - } & \text { De Contreras \& Pineda , } \\
& 2009 \\
\text { - } & \text { North \& Hargreaves, 2009; } \\
& \text { 2008; } 2003 \\
\text { - } & \text { Stack, Gundlach, \& Reeves, } \\
& 1994\end{array}$ \\
\hline $\begin{array}{l}\text { ARTÍCULOS DE } \\
\text { DESCRIPCIÓN Y } \\
\text { ANÁLISIS DE } \\
\text { INTERVENCIONES }\end{array}$ & $\begin{array}{ll}\text { - } & \text { Blood \& Zatorre, } 2001 \\
\text { - } & \text { Saarikallio, } 2011\end{array}$ & Schellenberg, 2004 & $\begin{array}{c}\text { North, Tarrant, \& Hargreaves, } \\
2004\end{array}$ \\
\hline LIBROS & $\begin{array}{ll}\text { - } & \text { Goleman, } 1996 \\
\text { - } & \text { Juslin, 2005 } \\
\text { - } & \text { Juslin \& Timmers, } \\
& 2010 \\
\text { - } & \text { Meyer, } 1956 \\
\text { - } & \text { North \& Hargreaves, } \\
& 2008 \\
\text { - } & \text { Sloboda \& Juslin, } \\
\end{array}$ & Peretz, 2010 & $\begin{array}{ll}\text { - } & \text { Csikszentmihalyi, } 2011 \\
\text { - } & \text { Dalia, 2008 } \\
\text { - } & \text { Trehub, Hannon, \& } \\
& \text { Schachner, 2010 }\end{array}$ \\
\hline
\end{tabular}




\begin{tabular}{|l|c|c|l|}
\hline & 2010 & & \\
& $\bullet \quad$ Storr, 2002 & & \\
\hline ACTAS DE & Jiménez, 2014 & & \\
CONGRESO & & Collins, 2012 & \\
\hline TESIS DOCTORAL & & & \\
\hline
\end{tabular}

Tabla 2: Clasificación de los estudios seleccionados

\section{RESULTADOS}

El análisis de los textos seleccionados se organiza en los siguientes apartados, resultado de las categorías temáticas que emergen de su estudio.

\section{Música y emoción}

Los estudios que componen este núcleo temático abordan la influencia de la música sobre la emoción y los efectos derivados de dicha influencia.

\section{Capacidad de la música para inducir emociones}

La influencia que la música ejerce sobre el estado anímico y comportamiento de la persona, es una cuestión que cuenta con una larga tradición. No obstante, es a partir de 1990 cuando las investigaciones centradas en esta temática comienzan a llevarse a cabo de forma sistemática (JUSLIN, 2003). Hoy en día existen numerosos estudios que respaldan la idea de que la música induce emociones en el ser humano (BlOOD \& ZATORRE, 2001; GABRIELSSON, 2002; KoELSCH, 2010, 2015; SlOBODA \& JuSLIN, 2010; STORR, 2002). La mayoría de ellos demuestran cómo, ante un estímulo musical, se activan áreas del cerebro correspondientes a la euforia y/o al placer (BLOOD \& ZATORRE, 2001). Esta estimulación cerebral va acompañada de una serie de reacciones físicas, entre las que destacan cambios en la presión sanguínea, cambios en el ritmo cardíaco y respiratorio, y respuesta galvánica de la piel (BLOOD \& ZATORRE, 2001; GUSTEMS, 2001).

Otros estudios afirman que la música ayuda a la regulación emocional (SAARIKALLIO, 2011), favoreciendo un estado emocional positivo (JuSLIN, 2005; KOELSCH, 2015). Según Sloboda y Juslin (2010), las emociones inducidas por la música suelen ser positivas. Bajo esta premisa se sustenta la musicoterapia, puesto que las emociones tienen una influencia directa tanto sobre el sistema nervioso vegetativo, como sobre el sistema hormonal e inmunológico (SORIA-URIOS, DUQUE \& GARCÍA-MORENO, 2011). Queda también demostrada la influencia de la música sobre el aspecto cognitivo, pues las funciones cognitivas que se requieren para su procesamiento favorecen el desarrollo de la memoria (SORIA-URIOS et al., 2011; VUILLEUMIER \& TROST, 2015), y sobre la mejora de la comunicación de personas con enfermedades crónicas (ANSDELLS \& PAVLICEVIC, 2005). Estos efectos son visibles a corto plazo, no quedando claro si perduran durante un período de tiempo más prolongado. Además, un aspecto interesante es el hecho de que es la emoción inducida por la música, y no la música en sí, la que favorece la recuperación (NORTH \& HARGREAVES, 2008).

Desde el ámbito de la psicología de la música se ha intentado dar respuesta a la cuestión de qué elementos musicales son los que inducen la emoción en la persona. Uno de los autores que más destacó en este campo fue Meyer, quien en su libro Emotion and Meaning in Music (1956), expresó la dificultad de saber con exactitud cuál es el estímulo musical que provoca la emoción en el oyente. Más recientemente, y siguiendo la línea de Meyer, Juslin (2005), argumenta que la respuesta emocional debe de ser producida por los elementos constantes que se dan dentro de la obra, tales como el tempo, la dinámica, los modos de ataque, la textura y un largo etcétera. En la actualidad, y en consonancia con la teoría de los afectos de la época barroca, la mayoría de los autores coinciden en una visión formalista de la expresión musical en la que tanto el compositor como el intérprete recurren a ciertos elementos musicales para producir en el oyente la emoción que pretenden (JiMÉNEZ, 2014; JusLin, 2003; MEYER, 1956; JuSLIN \& TiMMERS, 2010). 


\section{Función de la música en la sociedad}

Las funciones de la música en la sociedad están en relación directa con la capacidad de la música de inducir emociones. La música es utilizada en diferentes contextos a lo largo del día (Sloboda \& JusLin, 2010). Su uso puede ser de índole individual -cuando cada persona escucha un tipo de música para sí misma - o colectivo, cuando un tipo de música es seleccionado para inducir un comportamiento concreto a un grupo de personas. Un ejemplo de este último caso son los centros comerciales, donde suele utilizarse música rápida y fuerte para acelerar las compras de los clientes, o bien música lenta y placentera para fomentar una mayor permanencia en un lugar y suscitar un mayor consumo (NORTH \& HARGREAVES, 2008).

Según Sloboda (1985), otra de las funciones de la música es su potencial para ayudar al desarrollo de la humanidad, dada su capacidad para permitir que el ser humano exprese sus emociones, así como también para encontrar su identidad y mejorar las relaciones sociales. El sentimiento de pertenencia y de cohesión social favorece que los miembros de un mismo grupo desarrollen su propia identidad, lo que conduce a un mayor bienestar (KOELSCH, 2015; NORTH \& HARGREAVES, 2003). Otras funciones sociales de la música descritas por Koelsch (2015), y que incluyen experiencias en las que la música es compartida ya sea de forma pasiva (oyente) como activa (intérprete) son: a) la empatía, debido a que los distintos estados emocionales tienden a homogeneizarse dentro del grupo; b) la comunicación, pues es más sencillo expresarse a sí mismo a través de la música; o c) la cooperación, sobre todo si se ha de interpretar música dentro de un grupo, pues todos han de trabajar conjuntamente para lograr un fin común.

\section{Dificultad para medir las respuestas emocionales}

La relación que se establece entre estímulo musical y respuesta emocional no es de análisis sencillo. El hecho de concretar la naturaleza del estímulo musical que provoca respuestas emocionales es complejo; también lo es el mecanismo para medir la emoción inducida en la persona. Ésta depende de factores como el genético, el sociocultural, el histórico, el educacional (GUSTEMS \& CALDERÓN, 2004; ZATORE, 2003), el personal y el situacional (GABRILESSON, 2002; ROBINSON, 1995; SLOBODA \& JUSLIN, 2010). Dichos factores provocan que el comportamiento o respuesta psicológica ante un mismo estímulo musical sea distinto (JULIN \& TIMMERS, 2010). Otro potencial de la música incluye el despertar de emociones dolorosas que fueron reprimidas en algún momento y retenidas en el inconsciente, haciéndolas conscientes y permitiendo su curación a través de la expresión de las mismas (MEYER, 1956). Sobre esta cuestión, Goleman (1996) afirma que "la expresión artística puede despejar el camino para que los niños hablen de los terribles momentos vividos de un modo que sería imposible por otros medios" (p. 323).

Acorde con la literatura revisada en este apartado, la música afecta de forma directa a las emociones y, consecuentemente, a nuestro comportamiento. Esta característica la convierte en una herramienta fundamental para el conocimiento y regulación emocional.

\section{Música y neurociencia}

En este apartado se aborda la predisposición biológica del individuo hacia la música y cómo ésta afecta a la estructura y funciones del cerebro de los músicos.

\section{Predisposición natural del ser humano hacia la música}

Existen estudios que, si bien no son concluyentes, sugieren que el individuo tiene una predisposición biológica hacia la música (GRUHn, 2005; PERETZ, 2010; PERLOVSKY, 2010). Según Peretz (2002), el cerebro humano podría haber evolucionado para adaptarse al fenómeno musical por ser una herramienta eficaz para favorecer la cohesión del grupo. El aprendizaje musical comienza en el vientre de la madre y continua de forma informal después del nacimiento (GRUHN, 2005). Siguiendo con los postulados de Peretz (2002), la comprensión de los procesos de 
comunicación entre un recién nacido y su madre se producen a consecuencia de la entonación en el lenguaje. Se establece así una comunicación emocional. Por otro lado, según Gustems (2001), dado que el bebé está acostumbrado a escuchar sonidos en el vientre materno, la música podría favorecer que se sienta más confortable después de nacer. El autor afirma que existen estudios que demuestran que los niños prematuros que han de estar en incubadoras, presentan más resistencia y menos complicaciones de salud si escuchan música.

\section{Efectos de la práctica instrumental sobre el cerebro}

Si bien hasta el momento hemos hecho referencia tanto a oyentes como a intérpretes en relación a la influencia de la música en la persona, a lo largo de este apartado nos ocuparemos en exclusividad de los intérpretes, pues es el cerebro de éstos el que presenta mayores diferencias con respecto a aquéllos que no practican un instrumento musical (SORIA-URIOS et al., 2011).

El cerebro se encuentra dividido en dos partes iguales, las cuales conocemos como los hemisferios. Éstos se encuentran cruzados con respecto a las partes del cuerpo, es decir, el hemisferio derecho rige la parte izquierda del cuerpo y viceversa. Cada uno de los ellos cumple funciones biológicas diferentes (GUSTEMS \& CALDERÓN, 2004). Dichas funciones llevadas al ámbito de la interpretación musical se concretarían en las siguientes:

\begin{tabular}{|c|c|}
\hline HEMISFERIO IZQUIERDO & HEMISFERIO DERECHO \\
\hline 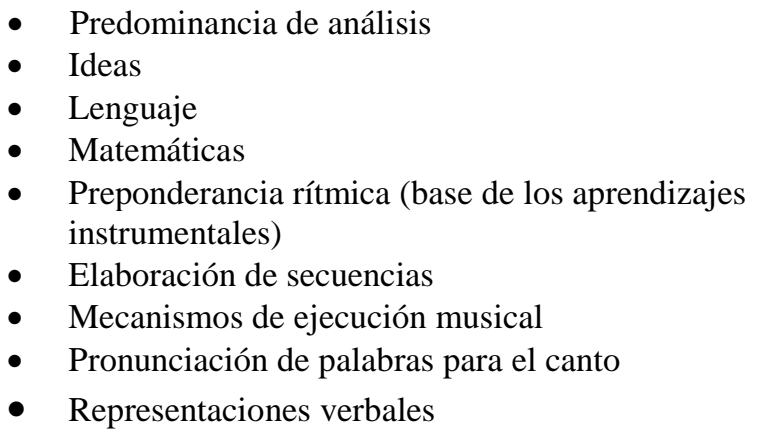 & $\begin{array}{l}\text { - } \quad \text { Predominancia de síntesis } \\
\text { - } \quad \text { Percepción del espacio } \\
\text { - } \quad \text { Percepción de las formas } \\
\text { - } \quad \text { Percepción de la música } \\
\text { - } \quad \text { Emisión melódica no verbal (intervalos, } \\
\text { - } \quad \text { Discriminación duración, etc.) } \\
\text { - } \quad \text { Función video-espacial } \\
\text { - Intuición musical } \\
\text { - Imaginación musical }\end{array}$ \\
\hline
\end{tabular}

Tabla 3: Localización de las funciones de la música en el cerebro (Lacárcel Moreno, 2003, pp. 216-217)

Cuando se participa de forma activa en la interpretación de un instrumento, se ponen en funcionamiento tanto la parte del cerebro correspondiente a la ejecución instrumental, como la correspondiente a la emocionalidad y expresividad, por lo que podemos decir que interpretar un instrumento requiere una actividad holística del cerebro (LACÁRCEL, 2003). Debido a esta mayor interacción de los dos hemisferios, el cerebro de los músicos presenta una mayor velocidad de transferencia interhemisférica (SORIA-URIOS et al., 2011).

Practicar un instrumento de forma habitual tendrá consecuencias en la estructura del cerebro y, consecuentemente, en nuestro comportamiento y habilidades. Esta afirmación se basa en que la estructura del cerebro -que hace referencia a la arquitectura cerebral - y las funciones cerebrales que determinan la forma en la que el cerebro procesa la información, envía mensajes y trabaja según la estructura cerebral-, están estrechamente relacionadas, de manera que al cambiar la estructura del cerebro también cambia la forma de enviar los mensajes y viceversa (CoLlins, 2012).

La práctica musical requiere de largos períodos de concentración, constancia, memorización de pasajes largos, aprendizaje de diferentes estructuras musicales, así como del desarrollo de la técnica y aprendizaje de las convenciones de expresión para transmitir las distintas emociones en una obra musical. Todas estas características tienen una consecuencia positiva sobre la cognición, sobre todo durante la niñez, que es cuando el cerebro se está desarrollando (SCHELLENBERG, 2004). Collins (2012) argumenta que los estudios demuestran que el entrenamiento musical facilita y hace más eficaz el aprendizaje de nueva información. La autora afirma que existe una relación entre alumnos que asisten a clases formales de música y un alto rendimiento académico. Además, existen estudios 
que han encontrado una asociación positiva entre el estudio de un instrumento y la memoria verbal, habilidad espacial, lectura, atención selectiva y matemáticas (SCHELLENBERG, 2004). Sin embargo, Schellenberg realiza una aportación interesante a esta relación. El autor considera que la práctica musical potencia la función cognitiva y, consecuentemente, produce un pequeño incremento en el CI y un mayor rendimiento académico; por otra parte, el autor señala que precisamente aquellos niños que asisten con regularidad a clases de música tienen habitualmente un alto CI. Además, tanto en este estudio como en Altenmuller, Gruhn, Parlitz y Liebert (2000), se hace referencia a la capacidad del cerebro para aplicar las redes neuronales desarrolladas a través de la práctica instrumental a otras áreas. Es lo que se denomina capacidad de transferencia (HALLAM, 2010).

Otra posible efecto del estudio de un instrumento sobre el cerebro es el incremento de la plasticidad cerebral (COLLINS, 2012, 2013; HoDGES, 2010). Se ha comprobado que el cerebro está constantemente cambiando y creciendo, por lo que, cuanto mayor es la plasticidad, más saludable será este órgano (COLLINS, 2012, p. 48). Este aspecto, al igual que la mayoría de las consecuencias de la práctica musical, se ve acrecentado si los estudios musicales comienzan a edades tempranas.

En resumen, a nivel neurocientífico, se halla un efecto global positivo en el cerebro a consecuencia de la práctica de un instrumento de forma habitual, y se señalan diferentes beneficios, fundamentalmente en el ámbito cognitivo.

\section{Música y conducta}

Hemos visto que la música tiene efectos sobre el cerebro generadas, principalmente, por la práctica instrumental activa. Esta categoría se centra en el análisis de las conductas derivadas de dichos efectos.

\section{En oyentes}

La música induce o potencia las emociones y, consecuentemente, el comportamiento que de ellas se deriva. En esta línea, la música influye en el comportamiento del individuo a través de los estados anímicos que estimula en ellos (NorTh et al., 2004; NorTH \& HARGREAVES, 2009). Podemos distinguir entre aquellos casos en los que la música es seleccionada por la persona de forma intencionada y aquellos en los que la música es un recurso utilizado por agentes externos con la intención de modificar la conducta de la persona, y poder así conseguir unos fines concretos.

En el primero de los casos, la música es seleccionada por la persona para cubrir unas necesidades que normalmente son de índole emocional. Estudios experimentales han demostrado que la música elegida por la persona es un hecho intencionado. De esta manera, si la persona necesita potenciar una mejora en su estado anímico, seleccionará una música que le induzca tranquilidad o seguridad; si, por ejemplo, tiene que hacer ejercicio físico, elegirá aquella que motive su ejecución (NORTH \& HARGREAVES, 2003). La percepción de la música escuchada depende de la situación de personal y, en ningún caso, será la música la que lleve a una persona a crear una situación que no esté en consonancia con su experiencia diaria. Esto cuestionaría afirmaciones como, según demuestran algunos estudios (STACK, GUNDLACH \& REEVES, 1994), que cierto tipo de música pueda inducir al suicidio. Acorde con North y Hargreaves (2009), es el individuo con pensamiento y conductas suicidas quien decide escuchar la música con la cual se identifica.

En el segundo de los casos, la música es utilizada para conseguir de forma intencionada fines concretos de un colectivo (TREHUB, HANNON \& SCHACHNER, 2010). Un ejemplo puede encontrarse en la pretensión de fomentar un mayor consumismo en los clientes de la cafeterías o inducir a los estudiantes a que mantengan bajos niveles de ruido en la clase (NORTH et al., 2004). En este caso la música no es elegida por el oyente, sino que este es sometido a sus influencias en diferentes contextos afectando, sin ser conscientes de ello, a su conducta. 


\section{En intérpretes}

La práctica de un instrumento musical requiere de una dedicación constante. A diferencia de otras disciplinas, al tratarse de una habilidad en la que hay que desarrollar un número considerable de competencias, además de la cognitiva, se necesita de un proceso lento y progresivo para la asimilación de los contenidos y destrezas que permitirán al intérprete poder ejecutar una obra con naturalidad (DE CONTRERAS \& PINEDA, 2009). Esta disciplina y rigor en el estudio tiene repercusiones en la conducta de la persona, las cuales podemos dividir en positivas y negativas. Algunas de estas características positivas propias de la conducta de un músico profesional son:

- Organización: es un requisito indispensable para un adecuada práctica instrumental (DE CONTRERAS \& PINEDA, 2009). La práctica musical requiere de una sabia organización y planificación, tanto del trabajo a realizar como del tiempo empleado. Un músico aprende a buscar diferentes estrategias acerca de cómo abordar su trabajo para tener mejores resultados en el menor tiempo posible.

- Perseverancia: en este punto es muy importante la motivación intrínseca del instrumentista. Si existe un alto deseo de interpretar con calidad una obra determinada, la probabilidad de éxito será mayor (CHUNG, 2010).

- Responsabilidad: por una parte, el instrumentista es pleno responsable de su instrumento ya que, en gran medida, de él depende el buen estado del instrumento. Al mismo tiempo, este también es responsable del tiempo que dedica al estudio del instrumento, así como de su agenda de ensayos y actuaciones (CASAS, 2001).

- Concentración: la práctica instrumental requiere la atención de un conjunto de aspectos que tienen lugar al mismo tiempo (afinación, duración de las notas, ritmo, frase, técnica de ejecución, expresión, entre otros). Esto demanda una gran concentración por parte del intérprete. Gracias a esta concentración, el intérprete es capaz de desarrollar la experiencia de entrar en estado de flujo (CSIKSZENTMIHALYI, 2011). El estado de flujo es definido como "el estado en el cual las personas se hallan tan involucradas en la actividad que nada más parece importarles; la experiencia, por sí misma, es tan placentera que las personas la realizarán incluso aunque tenga un gran coste, pro el puro motivo de hacerla" (p. 16).

- Disciplina: para poder conseguir los objetivos fijados se necesita disciplina y trabajo duro. El desarrollo de la fuerza de voluntad es importante si se quiere llegar a los objetivos propuestos y a vencer las dificultades crecientes con las que se encuentra un intérprete (CHung, 2010).

- Sentido del logro: para un instrumentista es importante establecer metas reales a conseguir a corto y largo plazo. Esto ayudará al intérprete a fomentar su motivación intrínseca al comprobar su autoeficacia. Los estudios demuestran que cuando el individuo experimenta que su trabajo ha sido efectivo, también potencia la confianza y la persistencia en la realización de la tarea. Se produce de esta manera la automotivación, la cual también es un requisito importante en el aprendizaje de un instrumento musical (DE CONTRERAS \& PINEDA, 2009; NORTH \& HARGREAVES, 2008).

- Por otra parte, la exigencia requerida por el instrumento, desarrolla en el músico una serie de tendencias conductuales que no siempre son beneficiosas. Para poderlas gestionar adecuadamente, la persona debe ser consciente de ellas. Siguiendo a Dalia (2008), algunas de estas conductas serían las siguientes:

- Individualismo: es una consecuencia de la cantidad de tiempo que el músico ha de pasar estudiando a solas.

- Pensamiento dicotómico: es habitual que el músico se mueva de un extremo a otro a la hora de juzgar su trabajo sin tener en consideración términos intermedios. 
- Competitividad: existe un fuerte sentimiento de competitividad entre los músicos desde muy temprana edad, produciéndose comparaciones que, con frecuencia, llevan a la frustración.

- Crítica, autocrítica y perfeccionismo: la autocrítica a la que se someten los músicos, a menudo va más allá de una evaluación necesaria para progresar y mejorar en su interpretación. Puede, en esencia, convertirse en un aspecto obsesivo, no tolerando cometer errores incluso en ensayos individuales y privados. Este mismo patrón de conducta se ve reflejado cuando escuchan a un compañero o colega pues, en lugar de disfrutar de la interpretación del mismo, con frecuencia su atención se centra en los posibles errores que puedan darse durante la interpretación.

Es deseable que el intérprete aprenda a paliar los indicados efectos negativos de la práctica musical para mejorar su bienestar tanto profesional como personal. Aprovechar el potencial que la práctica musical tiene para desarrollar las competencias emocionales favorecería la consecución de dicho fin.

\section{DISCUSIÓN}

\section{EI desarrollo de las competencias emocionales a través de la experiencia musical}

La literatura científica existente que vincula la música y las competencias emocionales, así como aquella que relaciona la música con la inteligencia emocional, es escasa. Por esta razón, y dada la estrecha vinculación entre los conceptos de inteligencia emocional y competencias emocionales (BISQUERRA \& PÉREZ, 2007), resulta de interés incluir en la literatura revisada estudios que aborden ambas temáticas. En el ámbito de la educación musical, son varios los estudios que defienden que las clases de música son una herramienta de utilidad para el desarrollo de las competencias emocionales (EDGAR, 2013; JACOBI, 2012). A pesar de que ninguno de los estudios analizados se basa en el análisis de una intervención, estos trabajos presentan una serie de sugerencias para la articulación de las competencias emocionales en el aula de música. En el campo experimental destaca el estudio de Schellenberg (2011), en el que el autor, en el análisis de la relación existente entre práctica instrumental e inteligencia emocional, concluye que no existe una correlación positiva entre ambos conceptos. Por otro lado, existen estudios en los que se abordan diversos aspectos relativos a las competencias emocionales con respecto a la música de una forma indirecta. Algunos de ellos establecen una relación entre práctica instrumental y comprensión de las emociones (SCHELLENBERG \& MANKARIOUS, 2012) y otros entre música y regulación emocional (SKANLAND, 2013; SAARIKALLIO, 2011).

Con base en la literatura revisada, se hace posible establecer una relación directa entre la música y las competencias emocionales que se concreta en la definición de una serie de competencias cuyo trabajo desde la educación musical puede resultar en beneficio de la persona:

1. Expresión de las emociones. La música es un excelente vehículo que favorece la expresión emocional de una forma que no podría darse a través de la palabra hablada. Esto se hace extensivo a las emociones que se encuentran reprimidas en el inconsciente (GOLEMAN, 1996).

2. Reconocimiento de las emociones. Partimos de que el oyente, aunque puede conmoverse por la música escuchada, no necesariamente tiene que sentir la emoción que la música expresa (STORR, 2002). Así pues, el oyente puede, por una parte, reconocer la emoción que una obra concreta expresa y, por otra parte, ser consciente de la emoción que esa obra suscita en él, incluyendo los procesos físicos que acompañan a la emoción que está sintiendo.

3. Regulación emocional. La música, por lo general, genera emociones placenteras en la persona, lo que favorece la regulación emocional (SAARIKALLIO, 2011).

4. Autoestima. Según North y Hargreaves (2008), cada persona posee diferentes autoconceptos, cada uno de ellos perteneciente a los distintos ámbitos que conforman su vida, y es la suma de ellos 
lo que definimos como identidad. La música favorece la construcción de dicha identidad lo cual, a su vez, repercute positivamente en la autoestima.

5. Empatía. El hecho de que varias personas compartan un espacio en el que la música está presente fomenta la empatía, pues este arte contribuye a que las emociones se intensifiquen y los sentimientos se coordinen (STORR, 2002).

6. Cooperación. Esta habilidad social es característica de la práctica musical, pues se desarrolla cuando un grupo de músicos debe aprender a trabajar en equipo, escucharse y compenetrarse para lograr satisfactoriamente un objetivo en común (CHUNG, 2010).

7. Escucha activa. El hecho de escuchar conscientemente una secuencia musical favorece la capacidad de escucha activa, aspecto importante para la adquisición de las habilidades sociales; este es un aspecto fundamental en el verdadero entendimiento entre personas (BISQUERRA, 2003).

8. Automotivación. En el caso del oyente, escuchar un determinado tipo de música puede ayudar a realizar una tarea concreta de forma más eficaz y fluida. En el caso del intérprete, este encuentra la fuerza para seguir adelante con las dificultades de la obra apoyándose en su automotivación, la cual es la capacidad para implicarse emocionalmente en actividades de diferentes ámbitos de la vida (BISQUERRA \& PÉREZ, 2007).

\section{Hacia una inclusión positiva de las competencias musicales en el aula de música}

La adquisición de las competencias emocionales resulta fundamental para desenvolverse satisfactoriamente en la vida, tanto en el ámbito profesional como en el personal (BISQUERRA \& PÉREZ, 2007; GolEMAN, 1998). La música, por su parte, está ligada a las emociones de forma natural, por lo que resulta útil para el desarrollo de dichas competencias (EDGAR, 2013; JACOBI, 2012; PellitTERI et al., 1999). A pesar de la relevancia de este campo de estudio, la literatura científica sistemática que vincula ambas áreas es escasa. Este estudio resulta de la necesidad de revisar esta literatura con el fin de extraer unas conclusiones generales en el ámbito de conocimiento. A la luz de los resultados de dicho análisis, se concluye que:

1. El área de estudio que vincula la música y el desarrollo de las competencias emocionales requiere de una mayor investigación sistemática, particularmente a través de intervenciones analizadas desde el campo de la educación musical.

2. Existe una relación positiva recíproca entre música y competencias emocionales, de forma que un ámbito refuerza los efectos beneficiosos que el otro ámbito ofrece y viceversa.

3. Los beneficios de la música en el trabajo y desarrollo emocional afectan tanto a oyentes como a intérpretes, siendo mayores para estos últimos. Esto es debido a que los intérpretes, además de pasar más tiempo en contacto con la música, deben desarrollar destrezas y actitudes propias de la interpretación instrumental que pueden servir como herramientas para un adecuado control emocional. Se destaca la escucha activa, la concentración, la disciplina, la responsabilidad, la constancia y la expresión de las emociones.

4. Los beneficios obtenidos por la práctica instrumental se incrementan en proporción al tiempo de dedicación y a la edad a la que se comience la práctica del instrumento, siendo estos mayores si se comienza el desarrollo musical a edades tempranas.

5. La música, además de favorecer el desarrollo cognitivo necesario para la articulación de las emociones -especialmente en el ámbito interpretativo-, favorece la conexión y concienciación de las propias emociones. La concienciación emocional es requisito básico para el desarrollo de las competencias emocionales.

6. Las competencias emocionales pueden ayudar a paliar los efectos negativos que la práctica de un instrumento musical puede producir en el intérprete, como son, por ejemplo, la individualidad, el perfeccionismo y la competitividad. 
A resultas de estas conclusiones, se establece que las enseñanzas musicales pueden mejorar significativamente, si se incluyen en el currículo, contenidos propios del desarrollo de las competencias. Estos contenidos pueden ser impartidos de forma transversal en cada una de las asignaturas que conforman el currículo de las enseñanzas musicales. Los contenidos propios de la música pueden, a su vez, servir de herramienta para trabajar ciertos aspectos emocionales. Este tipo de enseñanza integral puede favorecer la formación de buenos profesionales, incentivar el disfrute hacia la práctica musical y capacitar a las personas para desenvolverse con mayor competencia en actividades cotidianas. Esto supone un necesario replanteamiento del currículo y requiere de una imprescindible formación de los docentes en este ámbito de conocimiento. Todos estos aspectos precisan de una mayor investigación que posibilite la concreción temática en el ámbito de estudio.

Las conexiones que en este estudio se establecen entre la música y las competencias emocionales se basan de manera fundamental en los efectos que la música produce sobre la emoción, cognición y comportamiento del individuo. Este hecho, consecuencia de la citada escasa literatura científica en el área de estudio, hace que dichas relaciones no pueden considerarse como conclusivas. Al mismo tiempo, hoy en día no existe ninguna herramienta fiable para medir la respuesta emocional, e incluso los resultados de los estudios experimentales llevados a cabo no son concluyentes en su totalidad. Sin embargo, esta revisión propone servir de estímulo para, por una parte, reforzar la relación existente entre música y competencias emocionales y, por otra, animar a docentes de la música a conocer, reflexionar y poner en práctica propuestas tendentes hacia la inclusión de las competencias emocionales en la educación musical.

\section{REFERENCIAS BIBLIOGRÁFICAS}

ALTENMULLER, E., GRUHN, W., PARLITZ, D., \& LIEBERT, G. (2000). The impact of music education on brain networks: evidence from EEG-studies. International Journal of Music Education, 35(1), 47-53. http://doi.org/10.1177/025576140003500115

ANSDELLS, G. \& PAVLICEVIC, M. (2005). Musical companionship, musical community. Music therapy and the porves and value of musical communication. In Musical Communication (pp. 193213). New York: Oxford University Press.

BISQUERRA, R. \& PÉREZ, N. (2007). Las competencias emocionales. Educación XX1, 10, 61-82. http://doi.org/10.5944/educxx1.1.10.297

BISQUERRA, R. (2003). Educación emocional y competencias básicas para la vida. Revista de Investigación Educativa, 21(1), 7-43.

BLOOD, A. J. \& ZATORRE, R. J. (2001). Intensely pleasurable responses to music correlate with activity in brain regions implicated in reward and emotion. Psychology, 98(20), 11818-11823.

CASAS, M. V. (2001). ¿Por qué los niños deben aprender música? Colombia Médica, 32(4), 197204.

CHERNISS, C. (2000). Emotional Intelligence: What it is and Why it Matters. Pater presented at $15^{\text {th }}$ Annual Meeting of the Society for Industrial and Organizational Psychology, New Orleans, LA, 13-16 April, 2000.

CHUNG, B. (2010). Music making and the Well Termpered Life. Understanding Our Gift, 22(4), $3-5$.

COBB, C. D. \& MAYER, J. D. (2000). Emotional Intelligence: When integrating the concept of emocional intelligence into curriculum practice, educators need to understand the models, rely on solid research, and-as always-tread carefully. Educational Leadership, 14-18.

COLLINS, A. (2013). Music Education and the Brain: What Does It Take to Make a Change? 
Applications of Research in Music Education, 32(2), 4-10.

http://doi.org/10.1177/8755123313502346

COLLINS, A. M. (2012). Bigger, better brains: neuroscience, music education and the pre-service early childhood and primary (elementary) generalist teacher. The University of Melbourne.

CSIKSZENTMIHALYI, M. (2011). Fluir. Barcelona: Kairós.

DALIA, G. (2008). Cómo ser feliz si eres músico o tienes uno cerca. Madrid: Mundimúsica Ediciones.

DAMASIO, A. R. (2001). Emotion and the Human Brain. Annals of the New York Academy of Sciences, 935(1), 101-106. http://doi.org/10.1111/j.1749-6632.2001.tb03475.x

DE CONTRERAS, A. \& PINEDA, J. (2009). Reflexiones en cuanto a la organización de la práctica instrumental. Recuperado May 8, 2013, from http://musicalia-

digital.blogspot.com.es/2009/02/tecnicas-de-estudio-para-el-guitarrista.html

EDGAR, S. N. (2013). Introducing Social Emotional Learning to Music Education Professional Development. Applications of Research in Music Education, 31(2), 28-36.

http://doi.org/10.1177/8755123313480508

FERNÁNDEZ-BERROCAL, P., BERRIOS-MARTOS, M. P., EXTREMERA, N. \& AUGUSTO, J. M. (2012). Inteligencia emocional: 22 años de avances empíricos. Behavioral Psychology/ Psicologia Conductual, 20(1), 5-13.

FERNANDEZ-BERROCAL, P. \& EXTREMERA-PACHECO, N. (2005). La Inteligencia Emocional y la educación de las emociones desde el Modelo de Mayer y Salovey. Revista Interuniversitaria de Formación de Profesorado, 19(3), 63-93.

GABRIELSSON, A. (2002). Emotion perceived and emotion felt: Same and different. Musicae Scientiae, 6(1), 123-148. http://doi.org/10.1177/102986490601000203

GARDNER, H. (1983). Frames of mind. New York: Basic Books.

GOLEMAN, D. (1995). Emotional Intelligence: Why it Can Matter More than IQ? London: Bloomsbury Publishing.

GOLEMAN, D. (1996). Inteligencia Emocional. Barcelona: Kairós.

GOLEMAN, D. (1998). La Práctica de la Inteligencia Emocional. Barcelona: Kairós.

GOLEMAN, D., BOYATZIS, R. \& MCKEE, A. (2002). El líder resonante crea más. Barcelona: Plaza y Janés.

GRUHN, W. (2005). Children need music. International Journal of Music Education, 23(2), 99101. http://doi.org/10.1177/0255761405052400

GUSTEMS, J. (2001). L'entorn sonor, un element educatiu de primer ordre. Temps d'Educació, (25), 77-88.

GUSTEMS, J. \& CALDERÓN, C. (2004). No t' emocionis ... Escolta ! L' ús de la música en 1' educació emocional. Revista Catalana de Pedagogia, 3, 331-347.

HALLAM, S. (2010). The power of music: Its impact on the intellectual, social and personal development of children and young people. International Journal of Music Education, 28(3), 269289. http://doi.org/10.1177/0255761410370658

HODGES, D. A. (2010). Can neuroscience help us do a better job of teachin music? General Music Today, 23(2), 3-12.

JACOBI, B. S. (2012). Opportunities for Socioemotional Learning in Music Classrooms. Music 
Educators Journal, 99(2), 68-74. http://doi.org/10.1177/0027432112459082

JIMÉNEZ, F. (2014). Las Competencias Básicas. La Competencia Emocional. In La Música: Productora y Canalizadora de Emociones en el Primer Ciclo de Educación Infantil. Experiencia en un Centro de Educación Infantil (pp. 237-248). Facultad de Educación de Ciudad Real.

JUSLIN, P. N. (2003). Five Facets of Musical Expression: A Psychologist's Perspective on Music Performance. Psychology of Music, 31(3), 273-302. http://doi.org/10.1177/03057356030313003

JUSLIN, P. N. (2005). From mimesis to catharsis: expession perception, and induction of emotion. In Musical Communication (pp. 85-115). New York: Oxford University Press.

JUSLIN, P. N. \& TIMMERS, R. (2010). Expression and Communication of Emotion in Music Performance. In Handbook of Music and Emotion (pp. 453-489). Oxford: Oxford University Press.

KOELSCH, S. (2010). Towards a neural basis of music-evoked emotions. Trends in Cognitive Sciences, 14(3), 131-137. http://doi.org/10.1016/j.tics.2010.01.002

KOELSCH, S. (2015). Music-evoked emotions : principles, brain correlates , and implications for therapy, Annals of the New York Academy of Sciences., 1337 (1), 193-201. doi: 10.1111/nyas.12684 Ann.

KOSMAN, A. L. (1980). Being properly affected. Virtues and feelings in Aristotle's Ethics. In Essays on Aristotle's Ethics (pp. 103-116). Berkeley: University of California Press.

LACÁRCEL, J. (2003). Psicología de la música y emoción musical. Educatio Siglo XXI, (20-21), $213-226$.

MAYER, J. D. \& SALOVEY, P. (1997). What is emotional intelligence? In Emotional development and emotional intelligence: Educational implications (pp. 3-31). New York: Basic Books.

MEYER, L. B. (1956). Emotion and Meaning in Music. Chicago: The University of Chicago Press. MOLERO, C., SAIZ VICENTE, E., \& ESTEBAN MARTÍNEZ, C. (1998). Revisión histórica del concepto de Inteligencia: Una aproximación a ala Inteligencia Emocional. Revista Latioamericana de Psicología, 30(1), 11-30.

NORTH, A. C. \& HARGREAVES, D. J. (2003). Is music important? Psychologist, 16(8), 406-410. NORTH, A. C. \& HARGREAVES, D. J. (2009). The power of music. The Psychologist, 22(12), 1012.

NORTH, A. C.; TARRANT, M., \& HARGREAVES, D. J. (2004). The Effects of Music on Helping Behavior: A Field Study. Environment \& Behavior, 36(2), 266-275. http://doi.org/10.1177/0013916503256263

NORTH, A. \& HARGREAVES, D. (2008). The Social and Applied Psychology of Music. New York: Oxford University Press.

PELLITTERI, J., STERN, R., \& NAKHUTINA, L. (1999). Music: The Sounds of Emotional Intelligence. Voices from the Middle, 7(1), 25-29.

PERETZ, I. (2002). Brain specialization for music. The Neuroscientist, 8(4), 372-380. http://doi.org/10.1177/107385840200800412

PERETZ, I. (2010). Towards a Neurobiology of Musical Emotions. In Handbook of Music and Emotion (pp. 99-126). Oxford: Oxford University Press.

PERLOVSKY, L. (2010). Musical emotions: Functions, origins, evolution. Physics of Life Reviews, 7(1), 2-27. http://doi.org/10.1016/j.plrev.2009.11.001 
ROBINSON, J. (1995). The Expression and Arousal of Emotion in Music. The Journal of Aesthetics and Art Criticism, 52(1), 13-22.

SAARIKALLIO, S. (2011). Music as emocitonal self-regulations throught adulthood. Psychology of Music, 39(3), 307-327. DOI 10.1177/0305735610374894

SAARNI, C. (1997). Emotional competence and self regulation in childhood. En P. Salovey y D. J. Sluyter, (1997). In Emotional development and emotional intelligence (pp. 35-66). New York: Basic Books.

SAARNI, C. (2000). Emotional Competence. A developmenal Perspective. En R. Bar-On y J. D. Parker (Eds.). In The Handbook of Emotional Intelligence. Theory, Development, Assesment, and Application at Home, School, and in the Worlplace (pp. 68-91). San Francisco: Ca: Jossey-Bass.

SALOVEY, P. \& MAYER, J. (1990). Emotional Intelligence. Imagination, Cognition and Personality, 9, 185-211.

SALOVEY, P. \& SLUYTER, D. J. (1997). Emotional Development and Emotional Intelligence. Educational Implications. New York: Basic Books.

SASTRE MORCILLO, P. M. (2014). La Inteligencia Emocional en el Aprendizaje de los Alumnos de Enseñanzas de Régimen Especial en los Conservatorio de Música. DEDICA. Revista de Educaçao E Humanidades, 6, 187-198.

SCHELLENBERG, E. G. (2004). Music lessons enhance IQ. Psychological Science, 15(8), 511514. http://doi.org/10.1111/j.0956-7976.2004.00711.x

SCHELLENBERG, E. G. (2011). Music lessons, emotional intelligence, and IQ. Music Perception, 29(2), 185-194. DOI 10.1525/mp.2011.29.2.185

SCHELLENBERG, E. G. \& MANKARIOUS, M. (2012). Music training and emotion comprehension in childhood. Emotion, 12(5), 887-891. http://doi.org/10.1037/a0027971

SKANLAND, M. S. (2013). Everyday music listening and affect regualcion: The role of MP3 players. International Journal of Qualitative Studies on Health and Well-Being, 8(20595). DOI 10.3402/qhw.v8i0.20595

SLOBODA, J. A. (1985). The Musica Mind: The Cognitive Psychology of Music. New York: Oxford University Press.

SLOBODA, J. A. \& JUSLIN, P. N. (2010). At the interface between the inner and outer world: Psychological perspectives. In Oxford University Press (Ed.), Handbook of Music and Emotion (pp. 73-97). Oxford: Oxford University Press

SORIA-URIOS, G., DUQUE, P., \& GARCÍA-MORENO, J. M. (2011). Música y cerebro (II): Evidencias cerebrales del entrenamiento musical. Revista de Neurologia, 53(12), 739-746.

STACK, S., GUNDLACH, J., \& REEVES, J. L. (1994). The heavy metal subculture and suicide. Suicide and Life-Threatening Behaviour, 24, 15-23.

STORR, A. (2002). La música y la mente. El fenómeno autidivo y el porqué de las pasiones. Barcelona: Paidós.

TREHUB, S. E., HANNON, E. E., \& SCHACHNER, A. (2010). Perspectives on Music and Affect in the Early Years. In Handbook of Music and Emotion (pp. 645-668). Oxford: Oxford University Press.

TRUJILLO, M. M. \& RIVAS, L. A. (2005). Orígenes, evolución y modelos de inteligencia emocional. INNOVAR. Revista de Ciencias Administrativas Y Sociales, 15(25), 9-24.

VUILLEUMIER, P. \& TROST, W. (2015). Music and emotions: from enchantment to entrainment. 
Annals of the New York Academy of Sciences, 1337(1), 212-222. http://doi.org/10.1111/nyas.12676 ZATORRE, R. J. (2003). Music and the Brain. Annals Of The New York Academy Of Sciences, 999(1), 4-14. DOI 10.1196/annals.1284.001

Manuscrito recibido el 10/02/2016 y evaluado anónimamente. Aceptado para su publicación el 29/06/2016. 